\title{
HK DISTRIBUTION MODEL FOR ATMOSPHERIC TURBULENCE CHANNEL UNDER THE INFLUENCE OF POINTING ERRORS
}

\author{
Marko Smilić ${ }^{1 *}$, Stefan Panić ${ }^{1}$, Milan Savić ${ }^{1}$, Petar Spalević ${ }^{2}$, Dejan Milić ${ }^{3}$ \\ ${ }^{1}$ Faculty of Natural Sciences and Mathematics, University of Priština, Kosovska Mitrovica, Serbia. \\ ${ }^{2}$ Faculty of Technical Sciences, University of Priština, Kosovska Mitrovica, Serbia. \\ ${ }^{3}$ Faculty of Electronic Engineering, University of Niš, Niš, Serbia.
}

\section{ABSTRACT}

In this paper, HK statistical model is considered. The application of HK distribution in modeling atmospheric turbulence channel under the influence of strong and weak turbulences and all irradiance fluctuations are discussed. Expression for atmospherics turbulence channel model, pointing error models and path loss are given. Probability density functions (PDF) for both pointing error models (zero boresight and nonzero boresight) are calculated and graphically represented. Also, average bit-error-rate (BER) for zero boresight pointing error and nonzero boresight pointing error are calculated and graphically represented. Closed form expression for PDF and BER are given. Impact of zero boresight pointing error and nonzero boresight pointing error on transmission over atmospheric turbulence channel in FSO system is explained.

Key words: HK distribution model, FSO system, BER, PDF, Pointing error model.

\section{INTRODUCTION}

In last few years, atmospheric optical communications have becomes a subject of large interest. The reasons for these interests are simple because FSO system provide high security, high transmission capacity, low power and low cost characteristics in comparation with classical radio communications technologies (Heatley et al., 1998), (Jurado-Navas et al., 2012). For all of these advantages, FSO systems are the subject of bigger investigations and implementations. Atmospheric turbulence causes fluctuations in both the intensity and the phase of the received signal due to variations in the refractive index along the propagation path (Karp et al., 1988).

Many statistical models have been proposed to describe this fluctuation in both weak and strong fading regimes (Al-Habash et al., 2001). General Malaga distribution (Jurado-Navas et al., 2011) can be reduced to other distribution models such as GammaGamma distribution model (Harilaos et al., 2009), K distribution model (Jakerman, 1980), Lognormal distribution model (Zhu \& Kahn, 2002), HK distribution model (Destrempes et al., 2013), RiceNakagami distribution model (Tsiftsis et al., 2009). In addition to the turbulence, there are other scenario that affect the transmission link between transmitter and

receiver such as building sway and other constructions in urban areas. Weather conditions also affect on the quality and speed of transmission link (Andrews \& Philips, 1998). All of these lead to irradiance, misalignment (pointing error) between transmitter and receiver, scintillation and path loss. These scenarios affect on quality and speed of transmission link and channel capacity (Ansari et al., 2015).

In this paper, we considered influence of zero boresight pointing error and nonzero pointing error on transmission link over atmospherics channel under the strong and weak turbulences.

In addition to atmospheric channel model, we have presented pointing error models. Also, closed form expression for PDF and BER for HK model under the influence of zero boresight pointing error and nonzero boresight pointing error are calculated and graphically represented.

\section{SYSTEM MODEL}

Laser beams propagate along a horizontal path through a turbulence channel governed by an HK distribution in the presence of pointing errors (Peppas \& Mathiopoulos, 2015). The received photocurrent signal is related to the incident optical power by the 
detector responsivity $R$. It is assumed that the receiver integrates the photocurrent for each bit period and removes any constant bias due to background illumination (Farid \& Hranilovic, 2007). The received signal $y$ suffers from a fluctuation in signal intensity due to atmospheric turbulence and misalignment, as well as additive noise, and can be well modeled as:

$$
y=I(t) R P_{T}+n
$$

where $P_{T}$ is the transmitted intensity, $I(t)$ is the channel state, $y$ is the resulting electrical signal, and $n$ is signal-independent additive white Gaussian noise with variance.

The channel state $I(t)$ models the random attenuation of the propagation channel. In our model, $I(t)$ arises due to three factors: path loss $I_{l}(t)$, geometric spread and pointing errors $I_{p}(t)$, and atmospheric turbulence $I_{a}(t)$. The channel state can be formulated as:

$$
I(t)=I_{a}(t) I_{p}(t) I_{l}(t) .
$$

The HK distribution model can be obtained as a special case of Malaga distribution model. Malaga distribution model is given in (Tsiftsis et al., 2009) as:

$$
f_{I_{a}}\left(I_{a}\right)=A \sum_{k=1}^{\beta} a_{k} I_{a}^{\frac{\alpha+k}{2}-1} K_{\alpha-k}\left(2 \sqrt{\frac{\alpha \beta I_{a}}{\gamma \beta+\Omega}}\right) .
$$

For Malaga distribution model is valid $I=\left|U_{L}+U_{S}^{C}+U_{S}^{G}\right| e^{2 \chi}=Y X$ where are $X=e^{2 \chi}$ and $Y=\left|U_{L}+U_{S}^{C}+U_{S}^{G}\right|$ what is explained in (JuradoNavas et al., 2012). Setting by $\rho=0, X=\gamma$ and $\operatorname{Var}[G]=0$ we obtained HK distribution model where are $\quad \gamma=2 b_{0}(1-\rho)$. and $\Omega^{\prime}=\Omega+\rho 2 b_{0}+2 \sqrt{2 b_{0} \rho \Omega} \cos \left(\phi_{L}-\phi_{C}\right) \quad$ and $K_{v}(\cdot)$ is the modified Bessel function of the second kind and $v$ order. Using this conditions, we find that the HK distribution model is given:

$$
f_{I_{a}}\left(I_{a}\right)=A \sum_{k=1}^{\beta} a_{k} I_{a}^{\frac{\alpha+k}{2}-1} K_{\alpha-k}\left(2 \sqrt{\frac{\alpha \beta I_{a}}{2 b_{0} \beta+\Omega}}\right) .
$$

where

$$
A \triangleq \frac{2 \alpha^{\frac{\alpha}{2}}}{\left(2 b_{0}\right)^{1+\frac{\alpha}{2}} \Gamma(\alpha)}\left(\frac{2 b_{0} \beta}{2 b_{0} \beta+\Omega}\right)^{\beta+\frac{\alpha}{2}} .
$$

$$
a_{k} \triangleq\left(\begin{array}{c}
\beta-1 \\
k-1
\end{array}\right) \frac{\left(2 b_{0} \beta+\Omega\right)^{1-\frac{k}{2}}}{\Gamma(k)}\left(\frac{\Omega}{2 b_{0}}\right)^{k-1}\left(\frac{\alpha}{\beta}\right)^{\frac{k}{2}} .
$$

We have already explained what occurs due to the pointing errors. We consider two cases. In the first case, we consider zero boresight pointing error. Zero boresight pointing error is given:

$$
f_{I_{p}}\left(I_{p}\right)=\frac{g^{2}}{A_{0}^{g^{2}}} I_{p}^{g^{2}-1}, 0 \leq I_{p} \leq A_{0} .
$$

where $g=\omega_{z e q} / 2 \sigma_{s}$ is the ratio between the equivalent beam radius at the receiver $\omega_{z e q}$ and the pointing error displacement standard deviation at the receiver $\sigma_{s} . A_{0}=(\operatorname{erf}(v))^{2}$ is the fraction of the collected power where $v=\sqrt{\pi} a / \sqrt{2} \omega_{z}$ with $\operatorname{erf}(\cdot)$ denoting the error function, whereas the square of the equivalent beam width is given by:

$$
\omega_{z e q}^{2}=\omega_{z}^{2} \frac{\sqrt{\pi} e r f(v)}{2 v e^{-v^{2}}} .
$$

In the second case, we consider nonzero boresight pointing error. Nonzero boresight pointing error is given:

$$
f_{I_{p}}\left(I_{p}\right)=\frac{g^{2} e^{-\frac{s^{2}}{2 \sigma_{s}^{2}}}}{A_{0}^{g^{2}}} I_{p}^{g^{2}-1} I_{0}\left(\frac{s}{\sigma_{s}^{2}} \sqrt{\frac{-\omega_{z e q}{ }^{2} \ln \left(\frac{I_{p}}{A_{0}}\right)}{2}}\right) .
$$

where $s$ is the boresight displacement, $\sigma_{s}^{2}$ is the jitter variance at the receiver, $I_{0}(\cdot)$ is the modified Bessel function of the first kind with order zero.

$I_{l}$ denotes the atmospheric attenuation, which can be described by the exponential Beers-Lambert Law as:

$$
I_{l}(z)=e^{-\sigma z}
$$

where where $z$ denotes the propagation distance and $\sigma$ is the attenuation coefficient.

After defining both pointing error models, we can calculate the probability density functions. PDF is obtained by calculating the mixture of the two distributions presented above in equations (4) and (5) or equations (4) and (7).

$$
f_{I}(I)=\int_{0}^{\infty} f_{I \mid I_{a}}\left(I \mid I_{a}\right) f_{I_{a}}\left(I_{a}\right) d I_{a} .
$$

where $f_{I \mid I_{a}}\left(I \mid I_{a}\right)$ is the conditional probability given a turbulence state, $I_{a}$, and it is expressed as: 


$$
f_{I \mid I_{a}}\left(I \mid I_{a}\right)=\frac{g^{2}}{A_{0}^{g^{2}} I_{a}}\left(\frac{I}{I_{a}}\right)^{g^{2}-1}, 0 \leq I \leq A_{0} I_{a} .
$$

for zero boresight pointing error and:

$$
\begin{aligned}
& f_{I \mid I_{a}}\left(I \mid I_{a}\right)=\frac{g^{2} e^{-\frac{s^{2}}{2 \sigma_{s}^{2}}}}{A_{0}^{g^{2}} I_{a} I_{l}}\left(\frac{I}{I_{a} I_{l}}\right)^{g^{2}-1} \times \\
& \times I_{0}\left(\frac{s}{\frac{-\omega_{z e q}{ }^{2} \ln \left(\frac{I}{\sigma_{s}^{2}}\right)}{2}}\right), 0 \leq I \leq A_{0} I_{a} I_{l} .
\end{aligned}
$$

for nonzero boresight pointing error.

Substitunig expression (10) in (9) we get expression for PDF for zero boresight pointing error wich is represented as:

$$
\begin{aligned}
& f_{I}(I)=\frac{g^{2} A}{A_{0}^{g^{2}}} I^{g^{2}-1} \sum_{k=1}^{\beta} a_{k} \times \\
& \int_{\frac{I}{A_{0}}}^{\infty} I_{a}^{\frac{\alpha+k}{2}-1-g^{2}} K_{\alpha-k}\left(2 \sqrt{\frac{\alpha \beta I_{a}}{2 b_{0} \beta+\Omega}}\right) d I_{a} .
\end{aligned}
$$

After solving integral from equation (12) according to (Wolfram (07.34.21.0085.01)) where the modified Bessel function of the second kind, $K_{v}(\cdot)$ can be expressed as a special case of the Meijer G function, given by the following relationship (Prudnikov et al., 2003) we obtained closed form expression for PDF for zero boresight pointing error.

$$
\begin{aligned}
& f_{I}(I)=\frac{g^{2} A}{2} I^{-1} \sum_{k=1}^{\beta} a_{k}\left(\frac{\alpha \beta}{2 b_{0} \beta+\Omega}\right)^{-\frac{\alpha+k}{2}} \times \\
& G_{1,3}^{3,0}\left(\frac{\alpha \beta}{2 b_{0} \beta+\Omega} \frac{I}{A_{0}} \mid \begin{array}{c}
g^{2}+1 \\
g^{2}, \alpha, k
\end{array}\right) .
\end{aligned}
$$

Substitunig expression (11) in (9) we get expression for PDF for nonzero boresight pointing error wich is represented as:

$$
\begin{aligned}
& f_{I}(I)=\frac{g^{2} A e^{-\frac{s^{2}}{2 \sigma_{s}^{2}}}}{\left(A_{0} I_{l}\right)^{g^{2}}} I^{g^{2}-1} \sum_{k=1}^{\beta} a_{k} \times \\
& \int_{\frac{I}{A_{0} I_{l}}}^{\infty} I_{a}^{\frac{\alpha+k}{2}-1-g^{2}} K_{\alpha-k}\left(2 \sqrt{\frac{\alpha \beta I_{a}}{2 b_{0} \beta+\Omega}}\right) \times \\
& I_{0}\left(\frac{s}{\sigma_{s}^{2}} \sqrt{\frac{-\omega_{z e q}^{2}}{2} \ln \left(\frac{I}{A_{0} I_{a} I_{l}}\right)}\right) d I_{a} .
\end{aligned}
$$

After solving integral from equation (14) we obtained closed form expression for PDF for nonzero boresight pointing error.

$$
\begin{aligned}
& f_{I}(I)=\frac{2 \pi g^{2} A e^{-\frac{s^{2}}{2 \sigma_{s}^{2}}}}{\omega_{z e q}{ }^{2}} I^{g^{2}-1} \sum_{k=1}^{\beta} \frac{a_{k} I^{\frac{\alpha+k}{2}-1}}{\left(A_{0} I_{l}\right)^{\frac{\alpha+k}{2}} \sin (\pi(\alpha-k))} \times \\
& \sum_{p=0}^{p}\left\{\frac{\left(\frac{\alpha \beta I}{\left(2 b_{0} \beta+\Omega\right) A_{0} I_{l}}\right)^{p-\frac{\alpha-k}{2}}}{\Gamma(p-(\alpha-k)+1) p !}\left(-\frac{\omega_{z e q}{ }^{2}}{4\left(p+k-g^{2}\right)} e^{-\frac{\omega_{z q}^{2} s^{2}}{8\left(p+k-g^{2}\right) \sigma_{s}^{4}}}\right)\right.
\end{aligned}
$$$$
\left.\frac{\left(\frac{\alpha \beta I}{\left(2 b_{0} \beta+\Omega\right) A_{0} I_{l}}\right)^{p+\frac{\alpha-k}{2}}}{\Gamma(p+(\alpha-k)+1) p !} \times\left(-\frac{\omega_{z e q}{ }^{2}}{4\left(p+\alpha-g^{2}\right)} e^{-\frac{\omega_{z q}{ }^{2} s^{2}}{8\left(p+\alpha-g^{2}\right) \sigma_{s}^{4}}}\right)\right\} .
$$

The study of the Average BER of the HK probability distribution in the presence of misalignment fading is considered. First of all, we have defined expression for ABER over BFSK modulation format (Panić et al., 2013), (Stefanović et al., 2015), (Golubović et al., 2014), (Cvetković et al., 2013).

$$
P_{e}=\int_{0}^{\infty} \frac{1}{2} \operatorname{erfc}\left(\frac{P_{T} R}{\sigma_{N}} I\right) f_{I}(I)
$$

where $\operatorname{erfc}(\cdot)$ is related to the complementary error function. If we represented $\operatorname{erfc}(\cdot)$ as special case of MeijerG function acoording to (Prudnikov et al., 2003) and substituting in (16) along with expression (13), integral from (16) can be solved from relation (Wolfram (07.34.21.0013.01)). Closed form expression for zero boresight pointing error for ABER is given:

$$
\begin{aligned}
& P_{e}=\frac{2^{\alpha} g^{2} A\left(\frac{2 b_{0} \beta+\Omega}{\alpha \beta}\right)^{\frac{\alpha}{2}}}{32 \pi \sqrt{\pi}} \sum_{k=1}^{\beta} 2^{k}\left(\frac{2 b_{0} \beta+\Omega}{\alpha \beta}\right)^{\frac{k}{2}} a_{k} \times \\
& \times G_{7,4}^{2,6}\left(\frac{8 R^{2} P_{T}^{2} A_{0}^{2}}{\sigma_{N}^{2}}\left(\frac{2 b_{0} \beta+\Omega}{\alpha \beta}\right)^{2} \mid\right. \\
& \left.\mid \frac{1-g^{2}}{2}, \frac{2-g^{2}}{2}, \frac{1-\alpha}{2}, \frac{2-\alpha}{2}, \frac{1-k}{2}, \frac{2-k}{2}, 1\right) .
\end{aligned}
$$




\section{NUMERICAL RESULTS}

Capitalizing on presented expressions we have efficiently evaluated and graphically presented ABER over over BFSK modulation format for both zero and non-zero boresight pointing scenarios in the function of FSO propagation link parameters.

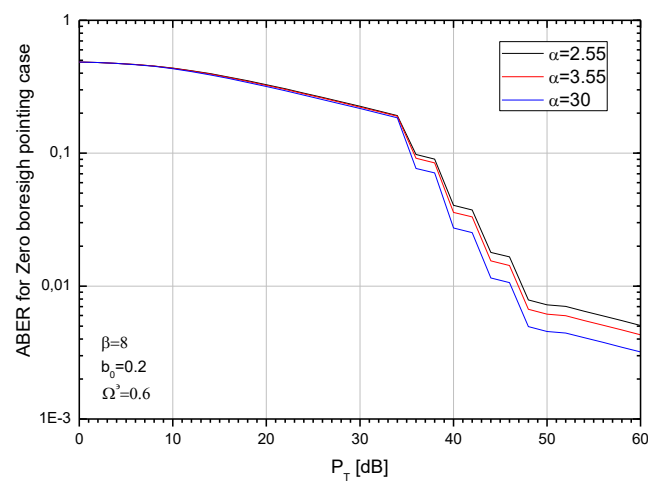

Fig. 1 ABER for Zero boresight pointing case.

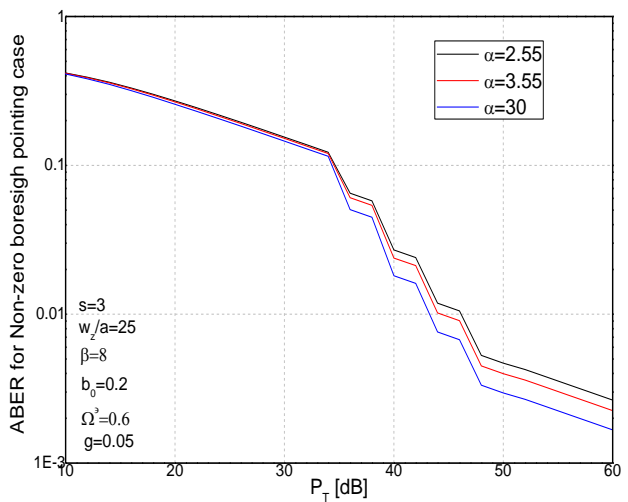

Fig. 2 ABER for Non-zero boresight pointing case.

It is visible from Figs 1-2 that better system performances are obtained for higher values of $\alpha$ parameter, i.e. for less severe scattering of FSO waves. $\alpha$ is a positive parameter related to the effective number of large-scale cells of the scattering process.

\section{CONCLUSION}

In this paper, two analytical closed-form representations for the ABER performance of an AOC system operating over a generalized turbulence in the presence of pointing errors are derived. Also, two analytical closed-form representations for the PDF are derived. Numerical results are graphically represented.

\section{REFERENCES}

Al-Habash, M.A., Andrews, L.C., \& Philips, R.L.. Mathematical model for the irradiance probability density function of a laser propagating through turbulent media. Optical Engineering, 40(8), pp. 1554-1562.

Ansari, I.S., Yilmaz, F., \& Alouini, M. 2015. Performance Analysis of Free-Space Optical Links Over Malaga (M) Turbulence Channels with Pointing Errors. IEEE Transactions on Wireless Communications, 15(1), pp. 91-102.

Andrews, L.C., \& Phillips, R.L. 1998. Laser Beam Propagation Through Random Media.SPIE.

Cvetkovic, A., Milic, D., \& Stefanovic, M. 2013. Outage Performance of Dual-Hop AF Relay Channels with Co-Channel Interferences over Rayleigh Fading. Wireless Personal Communications, 70(4), pp. 1993-2006.

Destrempes, F., Poree, J., \& Cloutier, G. 2013. Estimation Method of the Homodyned KDistribution Based on the Mean Intensity and Two Log-Moments. SIAM Journal Imaging Sciences, 6(3), pp. 1499-1530.

Farid, A.A., \& Hranilovic, S. 2007. Outage Capacity Optimization for Free-Space Optical Links With Pointing Errors. Journal of Lightwave Technology, 25(7), pp. 1702-1710. doi:10.1109/JLT.2007.899174.

Golubovic, A., Sekulovic, N., Stefanovic, M., Milic, D., \& Temelkovski, I. 2014. Performance Analysis of Dual-Branch Selection Diversity Receiver that Uses Desired Signal Algorithm in Correlated Weibull Fading Environment. Technical Gazette, 21(5), pp. 953-957.

Heatley, D.J.T., Wisely, D.R., Neild, I., \& Cochrane, P. 1998. Optical wireless:The story so far. IEEE Communications Magazine, 36(12), pp. 72-82. doi:10.1109/35.735881.

Jakerman, E. 1980. On the statistics of K-distributed noise. Journal of Physics A: Mathematical and General, 13(1), pp. 31-48.

Jurado-Navas, A., Garrido-Balsells, J.M., Paris, J.F., Castillo-Vázquez, M., \& Puerta-Notario, A. 2012. Impact of pointing errors on the performance of generalized atmospheric optical channels. Opt Express, 20(11), pp. 12550-62. pmid:22714243.

Jurado-Navas, A., Garrido-Balsells, J.M., Paris, J.F., \& Puerta-Notario, A. 2011. A unifying statistical model for atmospheric optical scintillation, Numerical Simulations of Physical and Engineering Processes., pp. 181-206.

Karp, S., Gagliardi, R., Moran, S., \& Stotts, L. 1988. Optical Channels.New York: Plenum.

Prudnikov, A.P., Brychkov, Y.A., \& Marichev, O.I. 2003. Integral and Series, 2nd ed.Moskva: Fizmatlit.

Panić, S., Stefanović, M., Anastasov, J., \& Spalević, P. 2013. Fading and Interference Mitigation in Wireless Communications.CRC Press. 
Peppas, K.P., \& Mathiopoulos, P.T. 2015. Free-Space Optical Communication With Spatial Modulation and Coherent Detection Over H-K Atmospheric Turbulence Channels. Journal of Lightwave Technology, 33(20), pp. 4221-4232.

Sandalidis, H.G., Tsiftsis, T.A., \& Karagiannidis, G.K. 2009. Optical Wireless Communications With Heterodyne Detection Over Turbulence Channels With Pointing Errors. Journal Of Lightwave Technology, 27(20), pp. 4440-4445.

Stefanovic, M., Anastasov, J., Cvetkovic, A., \& Djordjevic, G. 2015. Outage performance of dualhop relaying systems over extended generalized-K fading channels. Wireless Communications \& Mobile Computing, 15(18), pp. 2141-2149.

*E-mail: marko.smilic@pr.ac.rs
Tsiftsis, T.A., Sandalidis, H.G., Karagiannidis, G.K., \& Uysal, M. 2009. Optical wireless links with spatial diversity over strong atmospheric turbulence channels. IEEE Transactions on Wireless Communications, 8(2), pp. 951-957. doi:10.1109/TWC.2009.071318.

Wolfram... Retrieved

from: http://functions.wolfram.com/

Zhu, X., \& Kahn, J.M. 2002. Free-space optical communication through atmospheric turbulence channels. IEEE Transactions on Communications, 50(8), pp. 1293-1300. doi:10.1109/TCOMM.2002.800829. 